\title{
Corrigendum to "Low Body Mass Index Can Identify Majority of Osteoporotic Inflammatory Bowel Disease Patients Missed by Current Guidelines"
}

\author{
Ashish Atreja, ${ }^{1}$ Ashish Aggarwal, ${ }^{2}$ Angelo A. Licata, ${ }^{3}$ and Bret A. Lashner ${ }^{1}$ \\ ${ }^{1}$ Department of Gastroenterology, A-31, Digestive Disease Institute, Cleveland Clinic, 9500 Euclid Avenue, Cleveland, OH 44195, USA \\ ${ }^{2}$ Department of Internal Medicine, Medicine Institute, Cleveland Clinic, Cleveland, $\mathrm{OH} 44195$, USA \\ ${ }^{3}$ Department of Endocrinology, Center for Space Medicine, Bone Metabolic Institute, Cleveland Clinic, Cleveland, OH 44195, USA \\ Correspondence should be addressed to Ashish Atreja; atrejaa@ccf.org
}

Received 11 May 2017; Accepted 22 May 2017; Published 14 August 2017

Copyright (c) 2017 Ashish Atreja et al. This is an open access article distributed under the Creative Commons Attribution License, which permits unrestricted use, distribution, and reproduction in any medium, provided the original work is properly cited.

In the article titled "Low Body Mass Index Can Identify Majority of Osteoporotic Inflammatory Bowel Disease Patients Missed by Current Guidelines" [1], there was an error regarding the FRAX ${ }^{\circledR}$ tool, which should be clarified as follows.

The article notes: "In fact, the WHO fracture risk predictor model (FRAX) based on data derived from nine cohorts from Europe, North America, Asia, and Australia includes low BMI as an important clinical predictor to predict 10year probability of fracture [11]." However, the World Health Organization (WHO) did not develop, test, or endorse the FRAX tool or its recommendations [2]. The metabolic bone disease unit at the University of Sheffield that developed FRAX was a WHO Collaborating Centre from 1991 to 2010, but treatment guidelines must undergo a formal process before they can be endorsed by the WHO.

\section{References}

[1] A. Atreja, A. Aggarwal, A. A. Licata, and B. A. Lashner, "Low body mass index can identify majority of osteoporotic inflammatory bowel disease patients missed by current guidelines," The Scientific World Journal, vol. 2012, Article ID 807438, 6 pages, 2012.

[2] N. Ford, S. L. Norris, and S. R. Hill, “Clarifying WHO’s position on the FRAX ${ }^{\circledR}$ tool for fracture prediction," Bulletin of the World Health Organization, vol. 94, no. 12, p. 862, 2016. 


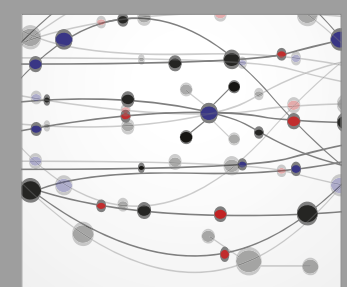

The Scientific World Journal
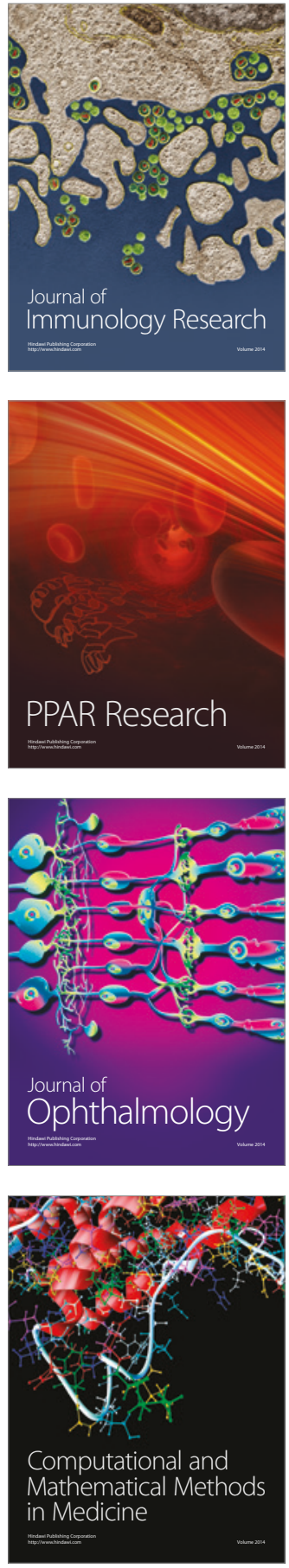

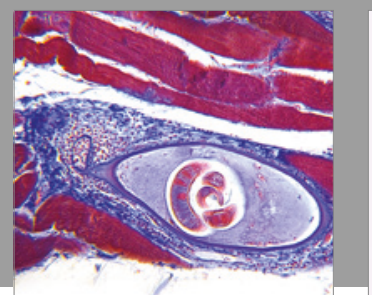

Gastroenterology Research and Practice
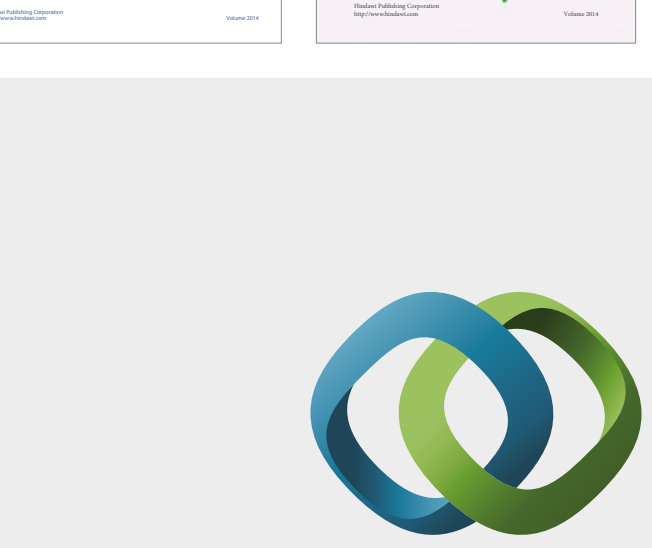

\section{Hindawi}

Submit your manuscripts at

https://www.hindawi.com
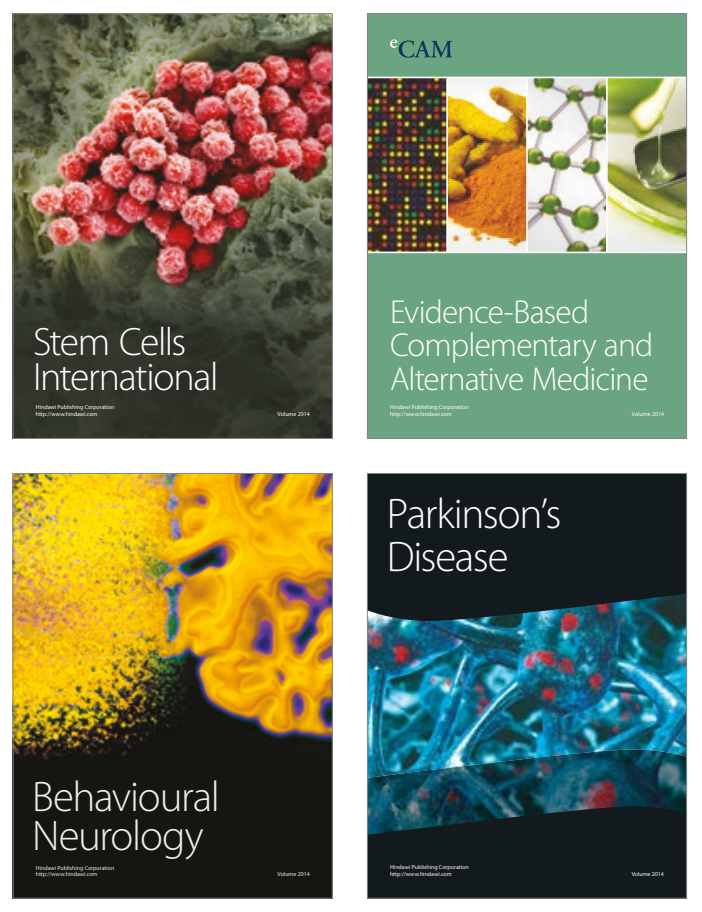
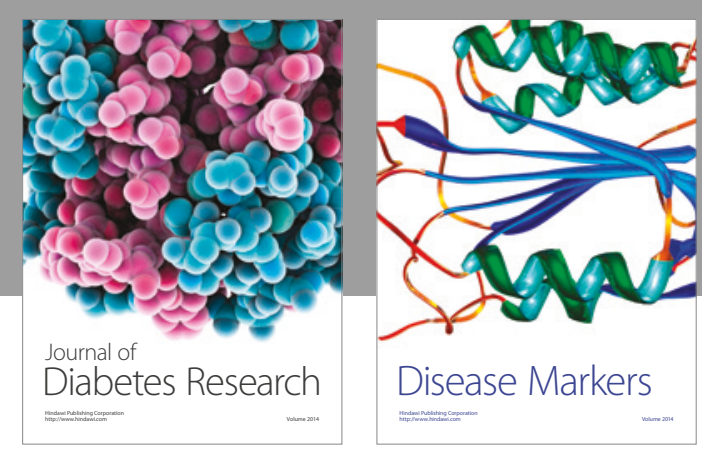

Disease Markers
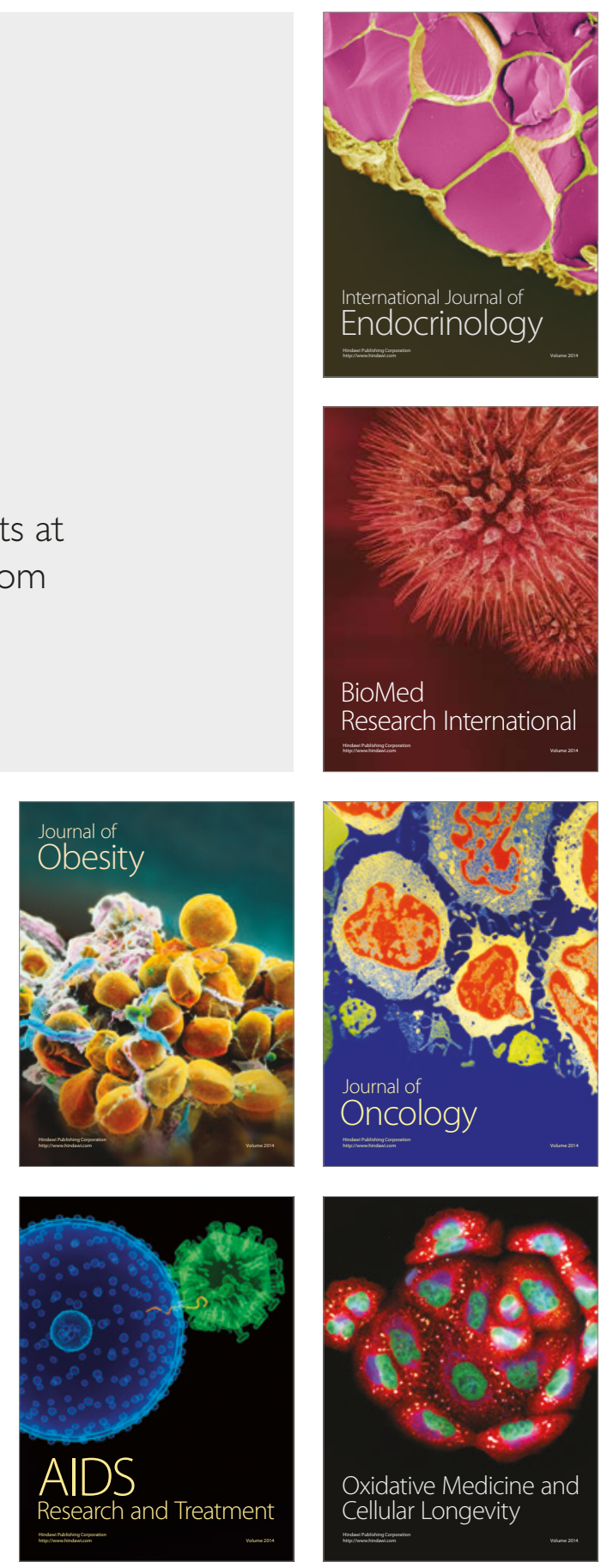